\title{
Morphological control and polarization switching in polymer dispersed liquid crystal materials and devices
}

\author{
K K RAINA*, PANKAJ KUMAR and PRAVEEN MALIK \\ School of Physics and Materials Science, Thapar Institute of Engineering and Technology, Patiala 147 004, India
}

\begin{abstract}
Liquid crystals dispersed in polymer systems constitute novel class of optical materials. The precise control of the liquid crystal droplet morphology in the polymer matrix is essentially required to meet the prerequisites of display device. Experiments have been carried out to investigate and identify the material properties and processing conditions required for the precise control of the droplet morphology of the dispersed liquid crystal systems. Polarization switching has been studied. Aligned liquid crystal dispersed systems showed higher polarization over unaligned ones.
\end{abstract}

Keywords. Polymer dispersed liquid crystals; nematic liquid crystal; ferroelectric liquid crystal; viscosity; droplet morphology; dichroic liquid crystal; contrast ratio; spontaneous polarization.

\section{Introduction}

In recent years, polymer dispersed liquid crystal (PDLC) composite materials have emerged as novel futuristic optical display materials because of their focus on extensive research, both fundamental and applied (Drzaic 1995; Valery and Kumar 1999; Lucchetti and Simoni 2000; Kikuchi et al 2002). They consist of micron and submicron sized liquid crystal (LC) droplets dispersed in a polymer matrix (usually thermo plastic). The PDLC based displays have a number of advantages over conventional liquid crystal display (LCD) devices, e.g. simple and minimal fabrication cost, flexible, high brightness, faster switching time and many others (Ono and Kawatsuki 1997; Karlsson and Konitor 1999; Simoni and Francescangeli 2000; Lehmann et al 2001).

LCDs using polymer dispersed ferroelectric liquid crystal (PDFLC) materials have been demonstrated to be atleast three orders of magnitude faster than the displays using nematic liquid crystals. It has also been reported that the contrast ratio and viewing angle of ferroelectric liquid crystal devices are superior to all other LC displays (Sumana and Raina 2005). Orientation structure inside the droplets, the droplet size and consequently the optical properties of these composite films are strongly dependent on polymer curing, viscosity, its molecular structure, magnitude of applied electric field, temperature and also the alignment of liquid crystal droplets in them. Both the threshold voltage and the switching speed of the resulting display are reported to decrease with increase in the droplet size (Drzaic 1995). Recently, dichroic PDLCs have

\footnotetext{
*Author for correspondence

(rainatietp@yahoo.com; kkraina@tiet.ac.in)
}

attracted significant interest among various research groups due to their technological applications (Malik et al 2003, 2004; Sumana and Raina 2005). The role of dyes in these systems is to allow an increase in the light absorption thus reducing remarkably the power necessary to induce nonlinear optical effects. PDLC films with dichroic dye dissolved in the liquid crystal (referred to as dichroic PDLC) possess both controllable absorbance and scattering as well. This combination can be used to produce high contrast displays (Drzaic et al 1989).

In this work, efforts have been made to study the material properties and processing conditions required for the precise control of liquid crystal droplet morphology of the dispersed systems. We have succeeded in aligning the ferroelectric liquid crystal droplets along the rubbing direction of the substrates to show better responses. The control of dye concentration to liquid crystal ratio led to better optical responses as well as the improved contrast of the device.

\section{Experimental}

Novel liquid crystal mixtures exhibiting nematic phase (E7, BL036) and ferroelectric phase (ZLI-3654) were obtained from E. Merck. UV curable polymers possessing different viscosities in the range 140-5000 CPS (NOA-73, NOA-65, NOA-63, NOA-68 procured from NORLAND, $\mathrm{NJ})$ were used for preparing PDLC films. E7 and BL036 have wide nematic liquid crystal temperature range whereas ZLI 3654 has wide ferroelectric smectic $\mathrm{C}$ temperature at room temperature. PDLC samples were prepared using polymerization induced phase separation (PIPS) technique. Liquid crystal to polymer ratio was taken as $30: 70 \mathrm{wt} / \mathrm{wt}$ to prepare polymer dispersed ferroelectric liquid crystal (PDFLC) samples. The sample cells essentially consisted 
of indium tin oxide (ITO) coated glass substrates pre-treated with the polyamide (nylon 6,6) solution by spin coating at $1400 \mathrm{rpm}$. These substrates were then heated in vacuum oven to remove traces of solvent, if any, to achieve a uniform film coating on the glass substrates. The treated glass substrates were rubbed uni-directionally using nylon cloth to induce planar alignment. The cell substrates were separated by about $10 \mu \mathrm{m}$ Mylar spacers to maintain the sample thickness. Spontaneous polarization studies were carried out using polarization current technique (Sumana and Raina 2004).

To obtain dichroic PDLC films, nematic liquid crystal mixtures were mixed with anthraquinone dye of different dye concentrations and then dispersed into the polymer material in $1: 1 \mathrm{wt} / \mathrm{wt}$ ratio. This homogenous mixture was heated to isotropic temperature in a vacuum oven and simultaneously shaken rapidly to ensure proper mixing. The PDLC material prepared by SIPS technique was sandwitched between the substrates and electrical connections applied for the measurements of physical parameters in presence of the electric field. The sample temperature was maintained at an accuracy of $0 \cdot 1^{\circ} \mathrm{C}$ in temperature programmer and hotstage (LINKAM model TP94 and THMS 600) interfaced to a computer. The microstructures of the samples were investigated through polarizing microscope (BX 51P) using thermal polarizing microscopy at a magnification of 10X. The details of the experimental procedures are reported elsewhere (Sumana and Raina 2005).

\section{Results and discussion}

\subsection{Droplet morphology of PDFLC films}

Figure 1(a-d) shows the micro-textures of unaligned PDFLC (NOA series + ZLI-3654) films in the order of increasing viscosity at a magnification of $10 \mathrm{X}$. It is seen that with increase of polymer viscosity, the droplet size of ferroelectric liquid crystal also increases. The droplet size has been measured by taking an average size of about 20 droplets in the polymer matrix. The variation in liquid crystal droplet size distribution and uniformity can be explained using Stoke's equation (Fuh and Caporaletti 1989) indicating that the liquid crystal droplet size and its uniformity is dependent on the sedimentation force as well as the polymer viscosity. As the polymer viscosity increases, the liquid crystal droplet size also increases. In aligned PDFLCs as shown in figure 2(a-d) respectively, it is seen that all the liquid crystal droplets are elongated along the rubbing direction. At lower viscosity, all the liquid crystal droplets are well aligned.

\subsection{Spontaneous polarization}

The spontaneous polarization $\left(P_{\mathrm{s}}\right)$ gives information about the internal structure of the mesophase and also about the quality of the phase separation since it is directly correlated
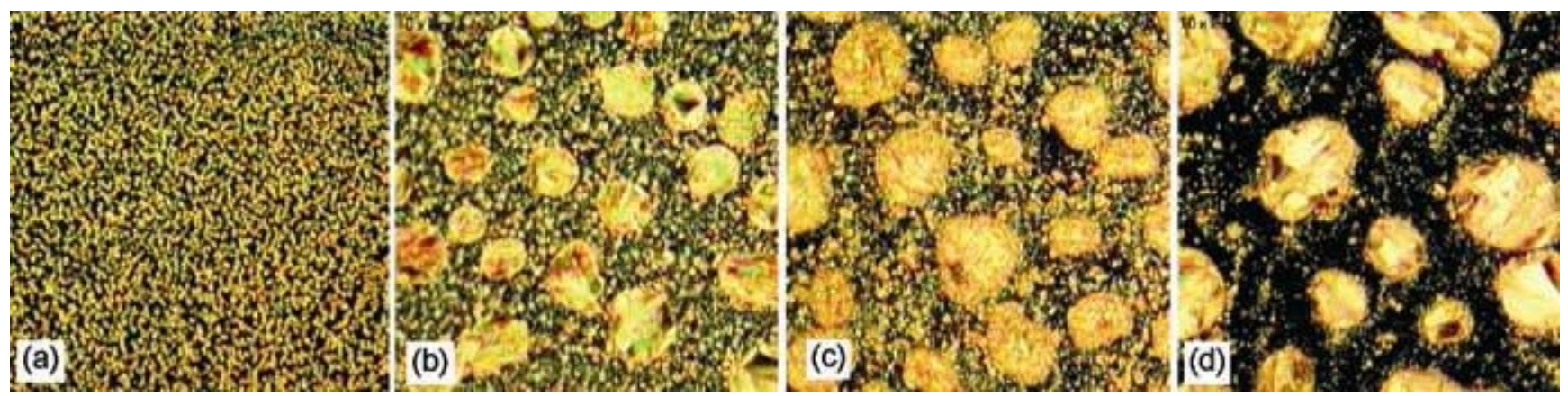

Figure 1. Micro-textures of the droplet morphologies of unaligned PDFLC film at 10X magnification under crossed polarizers at polymer viscosity: (a) 140 CPS, (b) 1000 CPS, (c) 2500 CPS and (d) 5000 CPS.
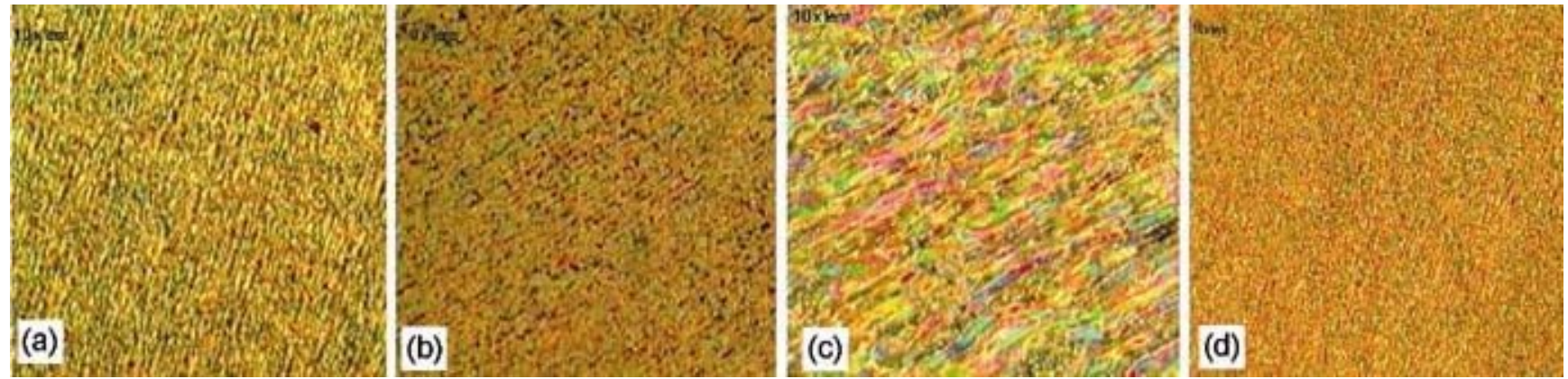

Figure 2. Effect of polymer viscosity on the alignment of liquid crystal droplets in aligned PDFLC films at $10 \mathrm{X}$ at polymer viscosity: (a) 140 CPS, (b) 1000 CPS, (c) 2500 CPS and (d) 5000 CPS. 


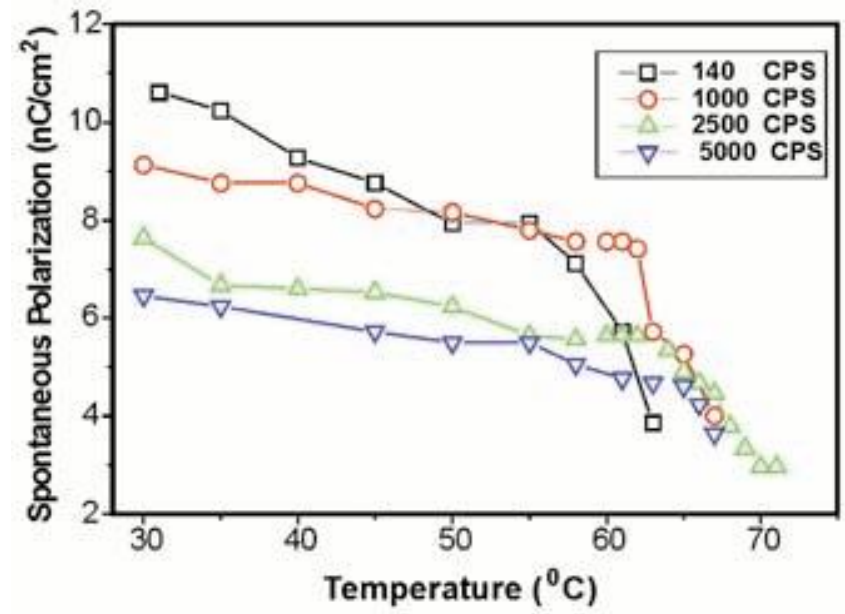

Figure 3. The effect of temperature on spontaneous polarization in aligned PDFLC films. to the fraction of switching molecules. Polarization switching responses were measured using field reversal technique (Sumana and Raina 2004). Spontaneous polarizations $\left(P_{\mathrm{s}}\right)$ of the aligned PDFLC composite films were calculated using NOA polymers of different viscosities. The spontaneous polarization as a function of temperature for UV cured PDFLC composite films at room temperature $\left(30^{\circ} \mathrm{C}\right)$ in presence of an electric field $\left(100 V_{\mathrm{PP}}, 100 \mathrm{~Hz}\right)$ is shown in figure 3 .

It is observed that with increasing temperature, spontaneous polarization decreases. At $\mathrm{SmC}^{*}-\mathrm{SmA}$ transition temperature $\left(T_{\mathrm{c}}^{*} A\right), P_{\mathrm{s}}$ suddenly drops to a very small value. Our results indicate that the PDFLC sample of lower polymer viscosity (NOA-73) shows higher $P_{\mathrm{s}}$ $\left(\sim 10.60 \mathrm{nC} / \mathrm{cm}^{2}\right)$ than the sample with higher polymer viscosity (NOA-68) $\left[P_{\mathrm{s}} \sim 6.45 \mathrm{nC} / \mathrm{cm}^{2}\right]$. Over the whole temperature range studied, we found that polarization

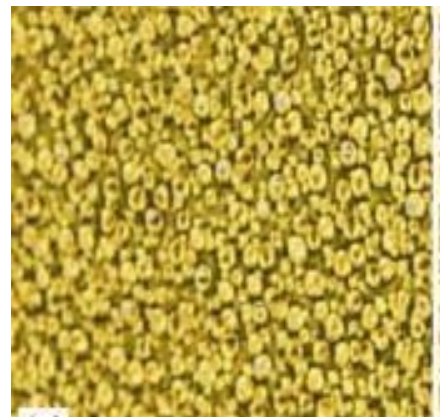

(a)
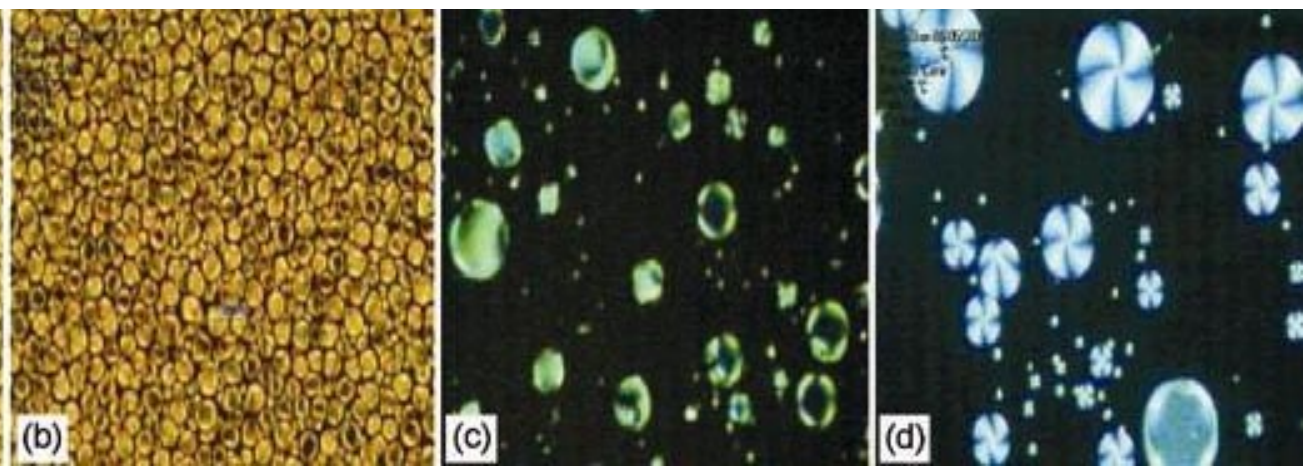

Figure 4. Droplet morphologies of dye doped polymer dispersed nematic liquid crystal (E7) with varying dye contents: (a) $0.125 \%$, (b) $0.25 \%$, (c) $0.5 \%$ and (d) $1 \%$.
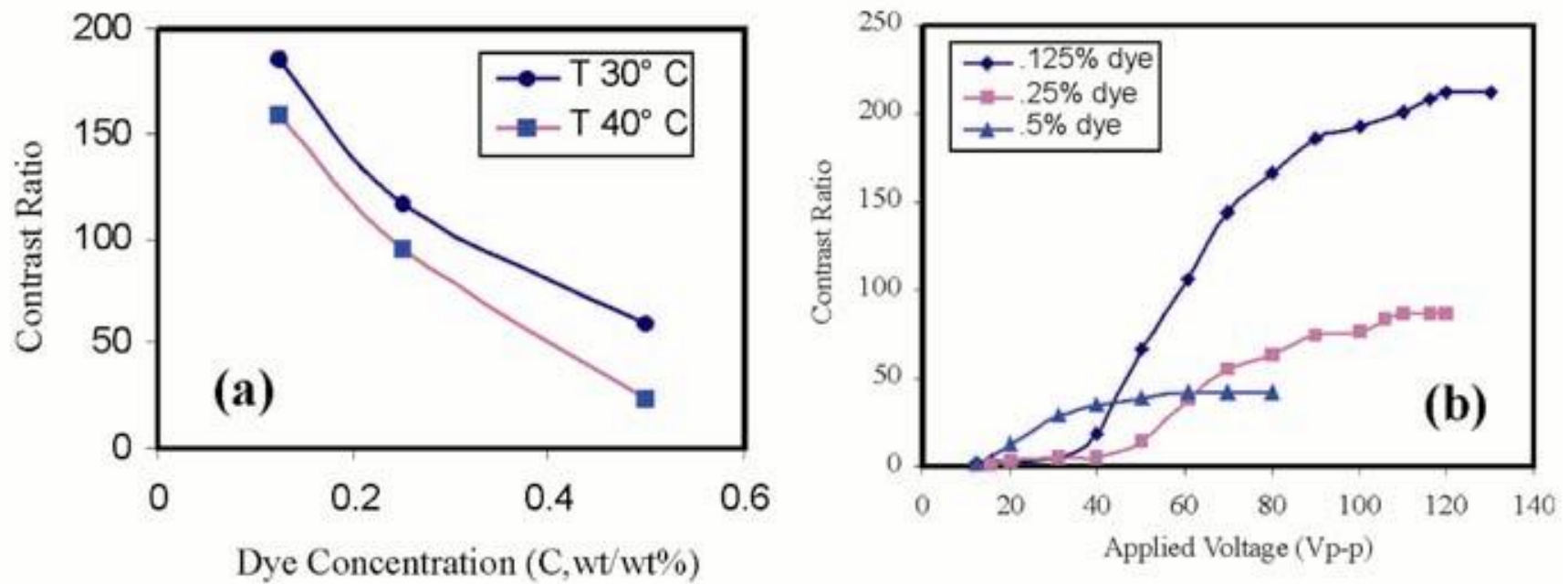

Figure 5. Dye dependence contrast ratio (a) at different temperatures and (b) as a function of applied voltage. 

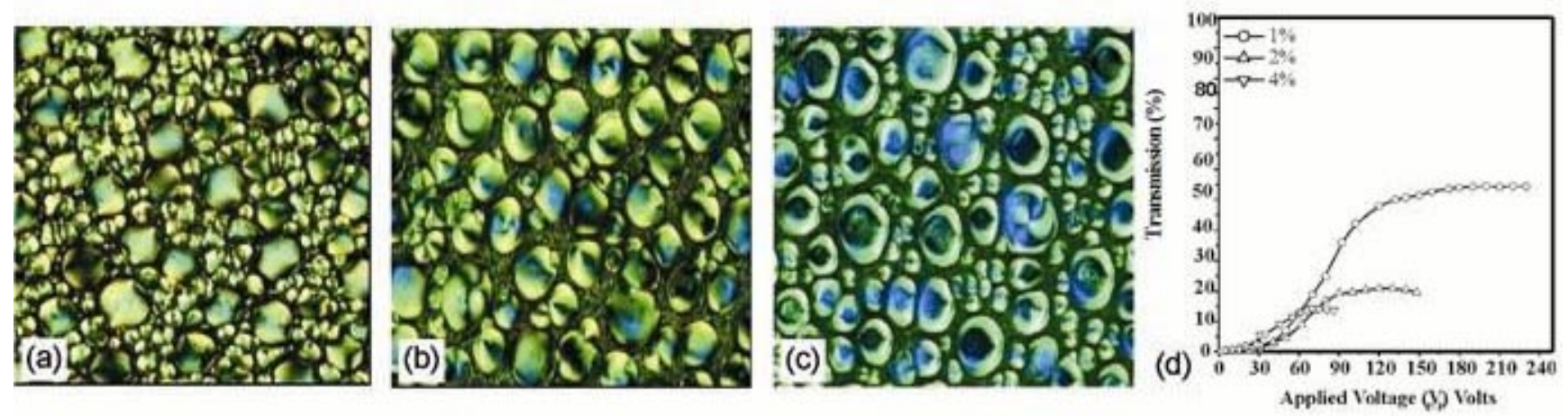

Figure 6. Influence of dye concentration: (a) 1\%, (b) 2\%, (c) 4\% on LC droplet morphology and (d) variation of applied voltage on optical transmission at different dye concentrations in nematic liquid crystal, BL036.

Table 1. Variation of the nematicisotropic transition temperature in dye doped PDLC.

\begin{tabular}{ll}
\hline E7 + NOA 65 & $T_{\mathrm{N}-\mathrm{I}}\left({ }^{\circ} \mathrm{C}\right)$ \\
\hline $0.125 \%$ dye & 59 \\
$0.25 \%$ dye & $57 \cdot 3$ \\
$0.5 \%$ dye & 55 \\
$1.0 \%$ dye & 45 \\
\hline
\end{tabular}

decreases but at the same time flexibility in the composite films has enhanced. A typical $P_{\mathrm{s}}$ dependence of PDFLC films as a function of polymer viscosity at different temperatures is shown in figure 3 . It is seen that $P_{\mathrm{s}}$ decreases with increase in polymer viscosity.

\subsection{Droplet morphology of dichroic PDNLC films}

Droplet morphology and phase behaviour of nematic E7 based dichroic polymer dispersed nematic liquid crystal (PDNLC) with $0.125 \%, 0.25 \%, 0.5 \%$ and $1 \%(\mathrm{wt} / \mathrm{wt})$ of dichroic dye, respectively was studied as shown in figure 4. It is seen that liquid crystal droplet size in the lower dye concentration $(\leq 0.25 \%)$ films is relatively smaller (in the range $\sim 5-15 \mu \mathrm{m}$ ) and more uniformly distributed forming spheroidal bipolar configuration (figure $4 \mathrm{~b}$ ) than higher dye concentration samples (figures $4 \mathrm{c}, \mathrm{d}$ ).

These include bipolar, axial, radial and toroidal (or collapsed) configurations. Studies performed on dye doped materials prove that a reduction in interfacial energy can cause an increase in the fraction of collapsed or toroidal droplets (Hall and Higgins 2003). We observed variation in the $T_{\mathrm{N}-\mathrm{I}}$ values as a function of dye concentration. The change in transition temperature from nematic to isotropic phase is shown in table 1 . We believe that this difference in the transition temperature might be due to some contaminations arising from dye and monomer molecules when dissolved in LCs (Smith 1993). The thermal contribution of light induced by absorption greatly enhanced by the presence of the dye, and its concert of dye in the mixture polymer/LC which causes a decrease in the clearing point.

\subsection{Contrast ratio}

Contrast ratio $\left(\mathrm{CR}=T_{\mathrm{ON}} / T_{\mathrm{OFF}}, T_{\mathrm{ON}}\right.$ is the maximum transmission and $T_{\mathrm{OFF}}$ the minimum transmission of a dichroic PDLC film) is an important measure of performance for any electro-optic display. Contrast ratio as a function of dye concentration and applied voltage is shown in figure 5(a, b) and it was observed that lower dye concentration PDLC film has much higher contrast ratio than the higher dye concentration PDLC films.

Optical textures of dichroic PDLC shows dominant bipolar configuration in comparison to other configurations (radial, axial) as in figure 6(a-c). The size of LC droplets was noticed in the range of $\sim 10-40 \mu \mathrm{m}$ in all the systems and their sizes are independent of electric field and temperature. The optical transmission as a function of applied voltage dependence at different dye concentrations is given in figure 6(d). It shows that nearly 50\% transmission can be achieved in $\sim 1 \%$ dye doped sample over $2 \%$ and $4 \%$ GHPDNLC samples. It can be concluded that $\sim 1 \%$ dye shows higher transmission than $4 \%$ sample. We believe that the higher absorption on $4 \%$ concentration leads to lower transmission of the guest host device. Thus $1 \%$ dye doped sample gives better transmission than higher dye concentration samples.

\section{Conclusions}

(I) In unaligned PDFLC films, liquid crystal droplet size increases with increasing polymer viscosity whereas in aligned sample, liquid crystal droplets elongated along the rubbing direction. The spontaneous polarization de- 
creases with increase in the polymer viscosity irrespective of the nature of alignment. The electro-optic responses of aligned PDFLC films are better than that of the unaligned PDFLC films.

(II) The transition temperature is lower for low dye concentration PDNLC films compared with a higher dye concentration PDNLC films. Our result indicates that lower dye concentration PDLC shows higher transmission, higher contrast ratio and better optical responses. It may find use in smart windows applications. Higher dye content results in a lower transmittance and a lower value of clearing temperature.

\section{Acknowledgements}

Authors wish to acknowledge DRDO and CSIR, New Delhi, India, for financial support. Thanks are due to $\mathrm{M} / \mathrm{s}$ Norland, NJ and E. Merck, UK, for providing the polymer and liquid crystal materials, respectively used in present studies.

\section{References}

Drzaic P S 1995 Liquid crystal dispersions (Singapore: World Scientific)

Drzaic P S, Wiley R and McCoy J 1989 J. Proc. SPIE 108041

Fuh A and Caporaletti O 1989 J. Appl. Phys. 665278

Hall J E and Higgins D A 2003 Polym. Mater. Sci. Eng. 88186

Karlsson M and Konitor L 1999 Mol. Cryst. Liq. Cryst. 231355

Kikuchi H, Yokota M, Hisakado Y, Yang H and Kajiyama T 2002 Nature Materials 164

Lehmann W, Skupin H, Tolksdorf C, Gebhard E, Zemta R, Kruger P, Losche M and Kremer F 2001 Nature 410447

Lucchetti L and Simoni F 2000 J. Appl. Phys. 883934

Malik P, Ahuja J K and Raina K K 2003 Curr. Appl. Phys. 3325

Malik P, Kumar P and Raina K K 2004 7th Int. conf. on optoelectronics, fibre optics and photonics, Cochin, India

Ono H and Kawatsuki N 1997 Opt. Lett. 221144

Simoni F and Francescangeli O 2000 Int. J. Polym. Mater. 45381

Smith G W 1993 Phys. Rev. Lett. 70198

Sumana G and Raina K K 2004 J. Appl. Polym. Sci. 94159

Sumana G and Raina K K 2005 Curr. Appl. Phys. 19588

Valery V and Kumar S 1999 Science 2831903 